\title{
The unusual nature of fingerprints and the implica- tions for easy-to-clean coatings
}

Bastian Stoehr ${ }^{a}$, Stuart McClure ${ }^{a}$, Alexander Höflich ${ }^{b}$, Mohammad Al Kobaisi ${ }^{c}$, Colin Hall ${ }^{a}$, Peter J. Murphy ${ }^{a}$ and Drew Evans $*^{a}$

${ }^{a}$ Future Industries Institute, University of South Australia, Mawson Lakes, SA 5095, Australia; email: drew.evans@unisa.edu.au

${ }^{\mathrm{b}}$ Fakultät Angewandte Chemie, Reutlingen University, Reutlingen, 72762, Germany

${ }^{c}$ Department of Chemistry and Biotechnology, Faculty of Science, Engineering and Technology, Swinburne University of Technology, Hawthorn, Victoria 3122, Australia 


\section{Supplementary}
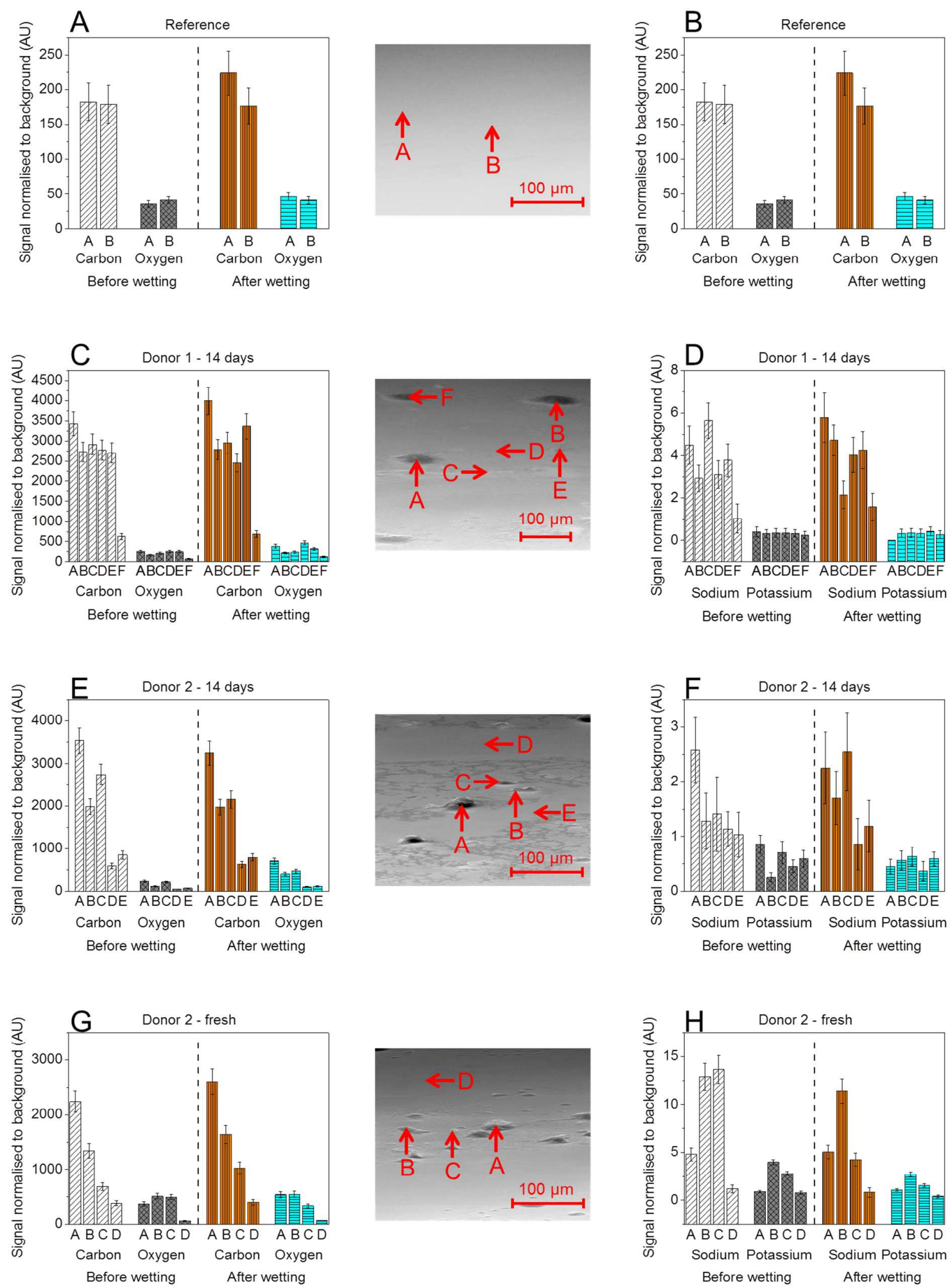

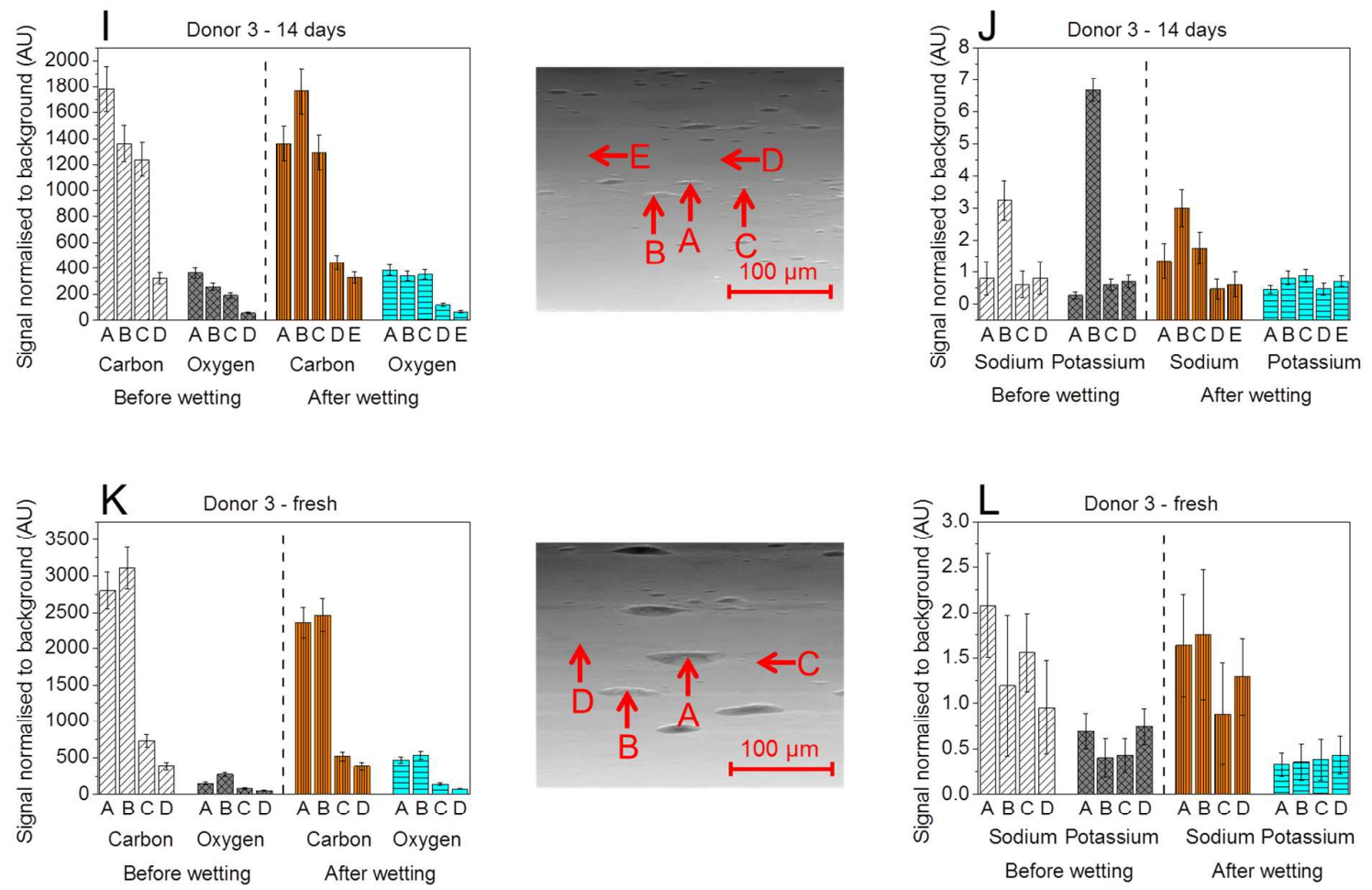

Fig. S1: Additional EDX analysis of selected elements from specific spots of real fingerprints obtained from three different donors. Numbers and letters below the columns in the graphs correlate to spots in the SEM images to show the exact spot locations. The data of the fresh fingerprint of Donor 1 is presented in the main body. 